\title{
Applications of recombinant Pichia pastoris in the healthcare industry
}

\author{
Daniel Weinacker ${ }^{1}$, Claudia Rabert $^{2}$, Andrea B. Zepeda ${ }^{1}$, Carolina A. Figueroa ${ }^{1}$, \\ Adalberto Pessoa $^{3}$, Jorge G. Farías ${ }^{1}$ \\ ${ }^{1}$ Departamento de Ingeniería Química, Facultad de Ingeniería, Ciencias y Administración, \\ Universidad de La Frontera, Temuco, Chile. \\ ${ }^{2}$ Departamento de Producción Agropecuaria, Facultad de Ciencias Agropecuarias y Forestales, \\ Universidad de La Frontera, Temuco, Chile. \\ ${ }^{3}$ Departamento de Tecnologia Bioquímico-Farmacêutica, Faculdade de Ciências Farmacêuticas, \\ Universidade de São Paulo, São Paulo, SP, Brazil.
}

Submitted: November 19, 2012; Approved: April 4, 2013.

\begin{abstract}
Since the 1970s, the establishment and development of the biotech industry has improved exponentially, allowing the commercial production of biopharmaceutical proteins. Nowadays, new recombinant protein production is considered a multibillion-dollar market, in which about $25 \%$ of commercial pharmaceuticals are biopharmaceuticals. But to achieve a competitive production process is not an easy task. Any production process has to be highly productive, efficient and economic. Despite that the perfect host is still not discovered, several research groups have chosen Pichia pastoris as expression system for the production of their protein because of its many features. The attempt of this review is to embrace several research lines that have adopted Pichia pastoris as their expression system to produce a protein on an industrial scale in the health care industry.
\end{abstract}

Key words: yeast, heterologous expression system, recombinant protein, production, scale up.

\section{Introduction}

\section{Recombinant protein industry}

Over the last few decades, geneticists have learned how to manipulate DNA to identify, move and place genes into a variety of organisms that are quite different from the source organism. Since the 1970s, the establishment and development of the biotech industry has improved exponentially, allowing the commercial production of industrial enzymes and biopharmaceutical proteins. Since many proteins are of immense commercial value, numerous studies have focused on finding ways to produce them efficiently and in a functional form (Macauley-Patrick et al., 2005; Porro et al., 2011). In 1980, the FDA approved for clinical use the recombinant insulin obtained from Escherichia coli, becoming the first recombinant pharmaceutical protein to enter the market. Since then, the biotechnology industry has grown substantially, and currently about $25 \%$ of commercial pharmaceuticals are biopharmaceuticals (Martínez et al., 2012). Recombinant protein production is a multibillion-dollar market, comprising biopharmaceuticals and industrial enzymes. Global sales for biopharmaceutical proteins reached US\$87 billion in 2008, and are expected to rise up to US\$169 billion in 2014. In 2009, out of the 151 approved recombinant biopharmaceutical products in the US and EU, 29 monoclonal antibodies contributed to more than $40 \%$ of the revenues, followed by vaccines, TNF blockers, hormones like insulins and erythropoietins (Porro et al., 2011). But to achieve a competitive production process is not an easy task. Any production process has to be highly productive, efficient and economic. The development of a new product or a new process usually begins with the choice of an appropriate production host; in this respect a comparative view on available expression hosts and their respective production processes is worthwhile (Porro et al., 2011).

Send correspondence to J.G. Farias. Departamento de Ingeniería Química, Facultad de Ingeniería, Ciencias y Administración, Universidad de La Frontera, Casilla 54D, Temuco, Chile. E-mail: jfarias@ufro.cl. 


\section{Expression systems}

Industrial biotechnology has traditionally employed numerous bacterial and eukaryotic organisms as production platforms. Many microbial products have indeed been available in the industrial biotechnology market for a long time now. With the advent of recombinant DNA technology, it has become possible, however, to introduce traits for the production of desired compounds into non-natural producers. Among microbial production, yeasts and bacteria share beneficial features as single cell growth, easy cultivation on cheap media and easy genetic manipulation (Porro et al., 2011). In general terms, bacteria have been considered to be the most efficient producers of heterologous proteins due to several reasons. However, standard prokaryotic systems have some limitations for production of human proteins. For example, bacteria are unable to perform some of the complex post-translational modifications (FerrerMiralles et al., 2009), which itself represents a limitation, since many proteins require further processing to become fully active. In particular glycosylations that are needed to ensure proper function and activity, by influencing proper charge, solubility, folding, serum half live of the protein, in vivo activity, correct cellular targeting and immunogenicity, among others, cannot be often be fully accomplished in bacterial systems (Walsh et al., 2006; De Pourcq et al., 2010; Martínez et al., 2012). On the other hand fungal expression systems, and in particular yeast, can grow in relatively cheap and defined media, decreasing the production costs. Besides, they are not so susceptible to contaminations and in addition, the yeast cells are less sensitive since the wall makes them more resistant to shear stress during the production process (De Pourcq et al., 2010; Martínez et al., 2012). In this case, the review will focus in one particular yeast; Pichia pastoris.

\section{Pichia pastoris}

The Pichia pastoris expression system is being used successfully for the production of various recombinant heterologous proteins (Rosenfeld et al., 1999; MacauleyPatrick et al., 2005; Li et al., 2007). Recombinant protein production in this yeast has several advantages over other eukaryotic and prokaryotic expression systems: (1) rapid growth rate, coupled with ease of high cell-density fermentation; (2) high levels of productivity in an almost protein-free medium; (3) elimination of endotoxin and bacteriophage contamination; (4) ease of genetic manipulation of well-characterized yeast expression vectors; (5) absence of known human pathogenicity in the spectrum of lytic viruses that prey on P. pastoris; (6) diverse posttranslational modifications that include polypeptide folding, glycosylation, methylation, acylation, proteolytic adjustment, and targeting to subcellular compartments; and (7) the ability to engineer secreted proteins that can be purified from growth medium without harvesting the yeast cells themselves (Li et al., 2007). All this features makes $P$. pastoris a useful system for both basic laboratory research and industrial manufacture. The fermentation can be readily scaled up to meet greater demands, and parameters influencing protein productivity and activity, such as $\mathrm{pH}$, aeration and carbon source feed rate, can be controlled (Higgins and Cregg, 1998). Compared with mammalian cells, Pichia does not require a complex growth medium or culture conditions, is genetically relatively easy to manipulate, and has a eukaryotic protein synthesis pathway (Macauley-Patrick et al., 2005). Also, protein purification from $P$. pastoris is straightforward. The secreted and soluble proteins can be directly recovered by clarification of the P. pastoris culture media by centrifugation. Samples can be concentrated and purified by subjecting the supernatant to ultrafiltration, precipitation, and/or adsorption/elution chromatography. The yield of secreted protein can be increased dramatically during fermentation by utilizing multistage processes that sequentially scale up yeast from small "starter cultures" in a non-fermenting fashion, increase biomass by feed-batch fermentation and, finally, induce the gene of interest (Cereghino et al., 2002).

The Pichia expression system has been widely used to produce a variety of different heterologous proteins. The wide range of promoters available, as well as selectable markers, secretion signals, methods for coping with proteases and a better understanding of glycosylation patterns, have given researchers diverse means to achieve the production of foreign proteins (Macauley-Patrick et al., 2005). Despite that diverse proteins are expressed in different types of industries, the focus in this review will be in the Health Care Industry. The idea is to explore several examples of how Pichia pastoris is used as an expression system to produce different kind of products, like antibodies and vaccines. It is shown in Figure 1 the research lines that are covered in this review that used $P$. pastoris as an expression system for the healthcare industry.

\section{Health Care Industry}

\section{Insulin production for diabetes}

Nowadays, human insulin is produced as recombinant protein, using two major routes. One route involves the production of an insulin precursor in the form of inclusion bodies, using E. coli as expression host with subsequent solubilization and refolding procedures. The other route involves the utilization of yeast- based expression systems, leading to the secretion of a soluble insulin precursor (IP) into the culture supernatant. Both routes are economically viable. The methylotrophic yeast Pichia pastoris has emerged as a very useful expression host with superior features (Gurramkonda et al., 2010). A synthetic insulin precursor (IP)-encoding genem codon-optimazed for expression in P. pastoris, was cloned in frame with the Saccharomyces cerevisiae $\alpha$-factor secretory signal and integrated into the genome of $P$. pastoris strain X-33. The 


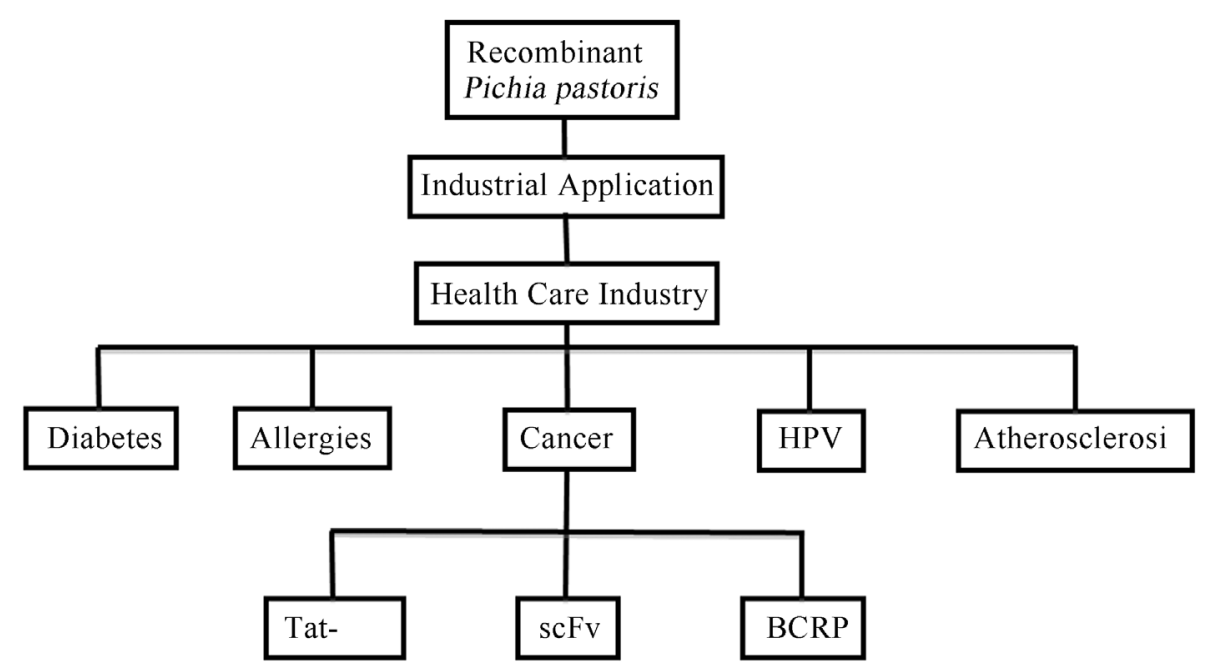

Figure 1 - Pichia pastoris applications in the health care industry. The diagram shows different lines of research in the healthcare industry that use recombinant $P$. pastoris as an expression system. HPV: Human Papillomavirus; Single-Chain Fragment Variable; BCRP: Breast Cancer Resistance Protein.

strain was grown to high-cell density in a batch procedure using a defined medium with low salt and high glycerol concentrations. Following batch growth, production of IP was carried out at methanol concentrations of 2 g. $\mathrm{L}^{-1}$, which were kept constant throughout the remaining production phase. This robust feeding strategy led to the secretion of $\sim 3$ gram IP per liter of culture broth (corresponding to almost 4 gram IP per liter of cell-free culture supernatant). Using immobilized metal ion affinity chromatography (IMAC) as a novel approach for IP purification, 95\% of the secreted product was recovered with a purity of $96 \%$ from the clarified culture supernatant. Finally, the purified IP was trypsin digested, transpeptidated, deprotected and further purified leading to $\sim 1.5 \mathrm{~g}$ of $99 \%$ pure recombinant human insulin per liter of culture broth (Gurramkonda et al., 2010). This aproach increased the efficiency of the insuline manufacture.

\section{Hypoallergenic derivatives of Ole e1 for vaccine development}

In Spain they used a new strategy for the design of a hypoallergenic derivative of Ole e1, the main allergen of olive pollen. It is one of the most important sources of allergy in Mediterranean countries and some areas of America, South Africa, Japan and Australia (Liccardi et al., 1996; White and Bernstein, 2003). By screening a cDNA library from birch pollen, the clone BB18, encoding the birch counterpart of Ole e1, was identified. In the study of Marazuela et al. (2012), BB18 was produced in Pichia pastoris as a recombinant protein and immunologically characterized. The well-established non-allergenic properties of BB18 were used to generate a genetic variant of Ole e1, named $\mathrm{OB}_{55-58}$, by site-direct mutagenesis of four residues (E55V56G57Y58) in an IgE/IgG epitope of Ole e1 by the corresponding ones in BB18 (SDSE). (Marazuela et al., 2012). Their results support the usefulness of BB18 for both epitope mapping of Ole e1 and engineering hypoallergenic derivatives of this allergen as for example the mutant OB55-58, which fulfills the requisites for a suitable hypoallergenic molecule. One of the final steps that this group need to do is to determine the best production and purification methods to became competitive in the hypoallergenic industry.

\section{Tat-p53 protein as a treatment for cancer}

P53 is an attractive target in molecular cancer therapeutics because of its critical role in regulating cell cycle arrest and apoptosis. It is one of the most commonly mutated tumor suppressor proteins in human cancers and plays a prominent role in recognition and repair of DNA damage, inhibition of malignant proliferation and cell transformation during cell growth (Moll et al., 2005). Although p53 has been transduced into cancer cells for therapy, the transmission of p53 into the cell membrane is limited due to the lack of the p53 ligand on membranes and its short half- life (Willis and Chen, 2002). A new approach to overcome this deficiency is to bind the protein or DNA molecule to a Tat construct which contains a protein transduction domain and therefore facilitates the penetration of tissue and cell membrane via molecular covalently connection as a fusion protein to transduce the membrane (Talos et al., 2005). Many experiments using this approach have been developed using the Escherichia coli expression system but none of them have yet achieved ideal protein yields. Yan et al. (2012) had better results using $P$. pastoris as an expression system. They have, for the first time, a high-level expression of recombinant human Tat-p53. Under the optimal conditions, $104 \mathrm{mg}$ Tat-p53 was recovered from 21 culture medium. Tat-p53 was purified by $>87 \%$ purity. The transduction of Tat-p53 fusion protein into tumor cells inhibited of tumor cell growth with evident apoptosis. 
Production of ScFv antibody fragments as potential markers for angiogenesis

Pichia pastoris expression system was used to produce functionalized single-chain antibody fragments (scFv) directed against the ED-B domain of the B-fibronectin (B-Fn) isoform (Marty et al., 2001). Single-chain Fv antibody fragments represent potential molecules for the targeted delivery of drugs, toxins or radionuclides and for coupling to liposomes for diagnostic and therapeutic applications. A scFv antibody fragment is an engineered antibody derivative that includes heavy- and light- chain variable regions joined by a peptide linker. In this case, the $\mathrm{scFv}$ antibody fragments recognizing the ED-B domain are potential markers for angiogenesis. Fibronectin $(\mathrm{Fn})$ is an extracellular adhesion molecule that mediates interactions between cells and extracellular matrix components. Fn is a glycoprotein composed of two subunits joined by disulfide bonds. The protein is involved in different biological processes, such as establishment and maintenance of morphology, cell migration, hemostasis and thrombosis, wound healing, and oncogenic transformation (Zardi et al., 1987). The B-Fn isoform with the ED-B oncofetal domain inserted by splicing is present in the stroma of fetal and neoplasic tissues and in adult and neoplastic blood vessels during angiogenesis but it is not detectable in mature vessels (Borsi et al., 1986; Kaczmarek et al., 1994). Therefore, the ED-B isoform of Fn represents a promising marker for angiogenesis in growing solid tumors. The group of Marty et al. (2001) cloned the sequence for the $\alpha$-ED-B scFv antibody fragments into the pPICZA $\alpha$ yeast vector containing a signal peptide sequence needed for protein secretion, a Zeocin resistance gene, and a flag-tag sequence that facilitates detection of the protein. They constructed four $\alpha$-ED$\mathrm{B} \mathrm{scFv}$ antibody fragments that were different in the number of cysteines and spacer length between the cysteines and the sequence of the scFv antibody fragment. The coupling efficiency of the fragments to small unilamellar liposomes of $50 \mathrm{~nm}$ mean diameter gave an average of $30 \mathrm{scFv}$ molecules linked to one liposome for three of the constructs (CM2, CM4, and CM5), whereas with the construct CM3 an average of 70 fragment molecules could be attached to one liposome. The majority of the purified protein eluted at $150 \mathrm{mM} \mathrm{NaCl}$ and the yield was $5-20 \mathrm{mg} / \mathrm{L}$ culture medium and the four $\mathrm{scFv}$ antibody fragments were concentrated to $0.5 \mathrm{mg} / \mathrm{mL}$ (Marty et al., 2001).

\section{Functional expression of the human breast cancer resistance protein}

The group of Mao et al. (2004) has established a $P$. pastoris expression system that is capable of producing breast cancer resistance protein (BCRP) at a high level. The system produced BCRP with functional characteristics very similar to those of the protein expressed in mammalian cells. BCRP is the second member of the subfamily $\mathrm{G}$ of the large $\mathrm{ABC}$ transporter superfamily. $\mathrm{BCRP}$ is a halftransporter (655 aa) consisting of only one NBD followed by one MSD with six predicted transmembrane a-helices (Doyle et al., 2003). Other studies suggest that BCRP may form homotetramers in the plasma membrane (Xu et al., 2004) and has a role in resistance to chemotherapeutic agents (Mao et al., 2004). They constructed the expression vector pHIL-BCRP-His10 containing BCRP cDNA and used it to transform the P. pastoris strain KM71. To facilitate future protein purification, a 10-histidine tag was attached to the $\mathrm{COOH}$-terminus of BCRP. The basal BCRP ATPase activity in the yeast membranes was approximately $40-80 \mathrm{nmol} \mathrm{P}_{\mathrm{i}} / \mathrm{min} / \mathrm{mg}$ protein, which can be modulated by known BCRP substrates and inhibitors. The $\mathrm{K}_{\mathrm{m}}$ and $\mathrm{V}_{\max }$ values of BCRP for $\left[{ }^{3} \mathrm{H}\right] \mathrm{E}_{1} \mathrm{~S}$ transport were $3.6 \pm$ $0.3 \mu \mathrm{M}$ and $55.2 \pm 1.6 \mathrm{pmol} / \mathrm{min} / \mathrm{mg}$ protein, respectively. This efficient and cost-effective expression system should facilitate large-scale production and purification of BCRP for further structural and functional analyses. All these data suggest that BCRP expressed in P. pastoris can form a stable transition-state complex with nucleotide and vanadate, which in turn indicates that BCRP expressed in P. pastoris is fully functional inhydrolyzing ATP (Mao et al., 2004).

\section{Vaccine production for Human Papillomavirus}

Human papillomavirus (HPV) infection is the most common sexually transmitted disease in the world and is related to the etiology of cervical cancer. The most common high-risk HPV types are 16 and 18 (Coimbra et al., 2011). To date, two prophylactic HPV vaccines are on the market, Gardasil $^{\mathrm{TM}}$ (Merck) and Cervarix ${ }^{\mathrm{TM}}$ (Glaxo-SmithKline). Studies have shown that these vaccines are safe, well tolerated and highly immunogenic. However, these vaccines are still inaccessible to the majority of the population in economically disadvantaged regions. The commercial HPV vaccines cost at least US\$360 for one person (three doses), an amount that is much higher than the annual per capita health expenditure of less developed countries. Therefore, there is a great need for other strategies for the production of cheaper HPV vaccines that could be provided by public health programs, allowing a greater penetration into these communities (Bazan et al., 2009). New vaccine strategies against HPV have shown that virus-like particles (VLP) of the major capsid protein (L1) induce efficient production of antibodies, which confer protection against the same viral type (Coimbra et al., 2011). It was recently demonstrated that $P$. pastoris could produce the HPV-16 L1 protein by using an episomal vector associated with the optimized L1 gene. However, the use of an episomal vector is not appropriate for protein production on an industrial scale. It is known that for large-scale production episomal vectors would not be advantageous since the transformed yeast can lose these vectors after successive mitotic divisions because they are not integrated into the genome and thus can be lost in the absence of selection pressure. The need of an- 
tibiotics for the maintenance of these clones raises the costs of production and may disagree with the rules imposed by regulatory agencies (Sreekrishna et al., 1997). Thus, to be able to produce the L1 protein on an industrial scale (vaccine production) it is important to obtain stable clones expressing the L1 gene and a manner to do this is to use integrative vectors. In addition, the use of an integrative vector may favor the integration of more than one copy of the heterologous gene, which in many cases increases protein production (Clare et al., 1991). In Coimbra et al., 2011 studies, they integrated the vectors into the $P$. pastoris genome and the results were positive for L1 gene transcription and protein production, both intracellularly and in the extracellular environment. The problem was that their results suggest a low yield of L1 recombinant protein, but they admitted that they could still do better in the yield optimization process. The achievement of stable clones containing the expression cassettes integrated in the genome may permit optimizations that could enable the establishment of a platform for the production of VLP-based vaccines (Coimbra et al., 2011).

\section{Development of a drug for Atherosclerosis}

Atherosclerosis, the major cause of many cardiovascular diseases, is a chronic inflammatory condition. Lipoprotein-associated phospholipase $\mathrm{A}_{2}\left(\mathrm{Lp}-\mathrm{PLA} \mathrm{A}_{2}\right)$ plays a crucial role in atherogenesis through the release of pro-inflammatory factors (Zalewski et al., 2005; Mello et al., 2011). Lp-PLA 2 is a secreted calcium-independent member of the phospholipase $\mathrm{A}_{2}$ superfamily. The majority of Lp-PLA 2 (about 70\%) in human plasma is associated with LDL and the remainder is associated with high-density lipoprotein (HDL). Lp-PLA 2 can hydrolyze oxidized low-density lipoprotein (ox-LDL) to generate lysophosphatidylcholine (lyso-PC) and oxidized fatty acids, both of which are pro-inflammatory and have been implicated in atherogenesis. Zhang et al. (2006) examined the feasibility of expressing and purifying Lp-PLA 2 through insect cell-baculovirus, yeast and $E$. coli expression systems, using vectors encoding Lp-PLA ${ }_{2}$ with and without an engineered Kozak sequence for high-level translation initiation. The insect cell-baculovirus system yielded high-level Lp$\mathrm{PLA}_{2}$ expression, with the recombinant protein accounting for up to $8 \%$ of total cellular proteins. The inclusion of the Kozak sequence further increased this expression up to $15 \%$ of total cellular proteins. Recombinant Lp-PLA $\mathrm{A}_{2}$ was expressed at a high level in the insect cell-baculovirus expression system, and was purified in an efficient two-step procedure. It showed good enzyme activity, and it was inhibited by SB435495 with an $\mathrm{IC}_{50}$ of $56.8 \pm 1 \mu \mathrm{M}$. The $P$. pastoris-based system showed a lower level of expression, but the recombinant Lp-PLA 2 could be purified rapidly and conveniently. $P$. pastoris-produced Lp-PLA 2 also exhibited correct enzyme activity, indicating that functional Lp$\mathrm{PLA}_{2}$ could be produced in eukaryotic expression systems.
P. pastoris-produced Lp-PLA 2 could be even more conveniently purified, as it required only a single nickel-chelating chromatography step for direct purification. In this case, the recombinant Lp-PLA2 fusion protein was designed to incorporate a C-terminal 6x His-tag to facilitate convenient purification by affinity chromatography. Because purification manipulation may decrease the enzyme activity and the protein yield, the purification method employed in this study gave an advantage over conventional multi-step purification method. In contrast, recombinant Lp-PLA2 expressed in E. coli showed no enzyme activity, indicating that the prokaryotic expression system likely failed to achieve correct protein processing, folding or post-translational modification. In this study, Zhang et al. (2006) describes two strategies for high-level expression or rapid purification of Lp-PLA2. Thus, large-scale production of recombinant Lp-PLA2 will facilitate further researches on this enzyme, and it may aid in the development of new drugs for the treatment of atherosclerosis. However, it does not suggest which one is better, because it depends what are the priorities of each research group.

\section{Conclusion}

It was found that different proteins production in $P$. pastoris for diseases like diabetes, cancer, HPV, and atherosclerosis achieved, in some cases, a better yield and functionality with lower cost than other expression systems. The proteins obtain usually have a direct effect on the diseases, but others acts like helper of another protein that has difficulties too penetrate in tissues and cell membranes by its own. Also, it was found that in most of these cases the goal of every laboratory was to produce cheaper, efficient and functional vaccines. Achieving these goals should facilitate large-scale production and purification of the protein of interest.

\section{Acknowledgments}

The authors knowledge the financial support of FAPESP (São Paulo Research Foundation, Brazil), CNPq (National Council for Scientific and Technological Development - Brazil) and CAPES (Coordination of Superior Level Staff Improvement, Brazil).

\section{References}

Bazan SB, de Alencar Muniz Chaves A, Aires KA, Cianciarullo AM, Garcea RL, Ho PL, (2009) Expression and characterization of HPV-16 L1 capsid protein in Pichia pastoris. Arch Virol 154:1609-1617.

Borsi L, Castellani P, Balza E, Siri A, Pellecchia C, De SF, Zardi L (1986) Large-scale procedure for the purification of fibronectin domains. Anal Biochem 155:335-345.

Cereghino GP, Cereghino JL, Ilgen C, Cregg JM (2002) Production of recombinant proteins in fermenter cultures of the yeast Pichia pastoris. Curr. Op Biotech 13:329-332. 
Clare JJ, Rayment FB, Ballantine SP, Sreekrishna K, Romanos MA (1991) High-level expression of tetanus toxin fragment $\mathrm{C}$ in Pichia pastoris strains containing multiple tandem integrations of the gene. Biotechnology 9:455-460.

Coimbra EC, Gomes FB, Campos JF, D'arc M, Carvalho JC, Mariz FC, Jesus ALS, Stocco RC, Beçak W, Freitas AC (2011) Production of L1 protein from different types of HPV in Pichia pastoris using an integrative vector. Braz J Med Biol Res 44:1209-1214.

De Pourcq K, De Schutter K, Callewaert N (2010) Engineering of glycosylation in yeast and other fungi: current state and perspectives. Appl Microbiol Biotechnol 87:1617-1631.

Doyle LA, Ross DD (20030 Multidrug resistance mediated by the breast cancer resistance protein BCRP (ABCG2), Oncogene 22:7340-7358.

Ferrer-Miralles N, Domingo-Espin J, Corchero JL, Vazquez E, Villaverde A (2009) Microbial factories forrecombinant pharmaceuticals. Microb Cell Fact 8:17.

Gurramkonda C, Polez S, Skoko N, Adnan A, Gäbel T, Chugh D, Swaminathan S, Khanna, N, Tisminetzky S, Rinas U (2010) Application of simple fed-batch technique to high-level secretory production of insulin precursor using Pichia pastoris with subsequent purification and conversion to human insulin. Microbial Cell Factories 9:31.

Higgins DR, Cregg JM (1998) Methods in Molecular Biology: Pichia Protocols. Humana: Totowa, NJ.

Kaczmarek J, Castellani P, Nicolo G, Spina B, Allemanni G, Zardi L (1994) Distribution of oncofetal fibronectin isoforms in normal, hyperplastic and neoplastic human breast tissues. Int. J Cancer 59:11-16.

Li P, Anumanthan A, Gao XG, Ilangovan K, Suzara VV, Düzgünes N, Renugopalakrishnan V (2007) Expression of Recombinant Proteins in Pichia Pastoris. Appl Biochem Biotechnol 142:105-124.

Liccardi G, D'Amato M, D'Amato G (1996) Oleaceae pollinosis: a review. Int Arch Allergy Immunol 111:210-217.

Macauley-Patrick S, Fazenda ML, McNeil B, Harvey LM (2005) Heterologous protein production using the Pichia pastoris expression system. Yeast 22:249-270.

Mao Q, Conseil G, Gupta A, Cole SPC, Unadkata JD (2004) Functional expression of the human breast cancer resistance protein in Pichia pastoris. Biochemical and Biophysical Research Communications. 320:730-737.

Marazuela EG, Hajek R, Villalba M, Barber D, Breiteneder H, Rodríguez R, Batanero E (2012) A non-allergenic Ole e 1-like protein from birch pollen as a tool to design hypoallergenic vaccine candidates. Molecular Immunology 50:83-90.

Martínez JL, Liu L, Petranovic D, Nielsen J (2012) Pharmaceutical protein production by yeast: towards production of human blood proteins by microbial fermentation. Curr Op Biotech 21:1-7.

Marty C, Scheidegger P, Ballmer-Hofer K, Klemenz R, A Schwendener RA (2001) Production of Functionalized Sin-
gle-Chain Fv Antibody Fragments Binding to the ED-B Domain of the B-isoform of Fibronectin in Pichia pastoris. Protein Expression and Purification 21:156-164.

Mello APQ, da Silva IT, Saes Parra Abdallab D, Teixeira Damascenoa NR (2011) Electronegative low-density lipoprotein: Origin and impact on health and disease. Atherosclerosis 215:257-265.

Moll UM, Wolff S, Speidel D, Deppert W (2005) Transcription independent pro-apoptotic functions of $\mathrm{p} 53$. Curr Opin Cell Biol 17:631-636.

Porro D, Gasser B, Fossati T, Maurer M, Branduardi P, Sauer M, Mattanovich D (2011) Production of recombinant proteins and metabolites in yeasts. Appl Microbiol Biotechnol 89:939-948

Rosenfeld SA, Nadeau D, Tirado J (1999) Production and purification of recombinant hirudin expressed in the methylotrophic yeast Pichia pastoris. Methods Enzymol 306:154-169.

Sreekrishna K, Brankamp RG, Kropp KE, Blankenship DT, Tsay JT, Smith PL (1997) Strategies for optimal synthesis and secretion of heterologous proteins in the methylotrophic yeast Pichia pastoris. Gene 190:55-62.

Talos F, Petrenko O, Mena P (2005) Mitochondrially targeted p53 has tumor suppressor activities in vivo. Cancer Res 65:9971-9981.

Walsh G, Jefferis R (2006) Post-translational modifications in the context of therapeutic proteins. Nat Biotechnol 24:12411252.

White JF, Bernstein DI (2003) Key pollens in North America. Ann. Allergy Asthma Immunol 91:425-435.

Willis AC, Chen X (2002) The promise and obstacle of p53 as a cancer therapeutic agent. Curr Mol Med 2:329-345.

Xu J, Liu Y, Yang Y, Bates S, Zhang JT (2004) Characterization of oligomeric human half $\mathrm{ABC}$ transporter ABCG2/BCRP/MXR/ ABCP in plasma membranes. J Biol Chem 279:19781- 19789.

Yan H, Liu N, Zhao Z, Zhang X, Xu H, Shao B, Yan W (2012) Expression and purification of human TAT-p53 fusion protein in Pichia pastoris and its influence on $\mathrm{HepG} 2$ cell apoptosis. Biotechnol Lett 34:1217-1223.

Zalewski A, Macphee C (2005) Role of lipoprotein-associated phospholipase $\mathrm{A}_{2}$ in atherosclerosis: biology, epidemiology, and possible therapeutic target. Arterioscler. Thromb Vasc Biol 25:923-931.

Zardi L, Carnemolla B, Siri A, Petersen TE, Paolella G, Sebastio G, Baralle FE (1987) Transformed human cells produce a new fibronectin isoform by preferential alternative splicing of a previously unobserved exon. EMBO J 6:2337-2342.

Zhang F, Dong Li, Cai M, Shen J, Wang Y (2006) Heterologous expression of lipoprotein-associated phospholipase A2 in different expression systems. Protein Expression and Purification 48:300-306.

All the content of the journal, except where otherwise noted, is licensed under a Creative Commons License CC BY-NC. 